RUNNING HEAD: FRAMEWORK FOR INSTRUCTIONS

Toward a Unified Framework for Research on Instructions and other Messages:

An Introduction to the Special Section on the Power of Instructions

Jan De Houwer, Sean Hughes, \& Marcel Brass

Ghent University, Belgium

In press. Neuroscience \& Biobehavioral Reviews.

mailing address: Jan De Houwer

Ghent University

Henri Dunantlaan 2

B-9000 Ghent

Belgium

email: Jan.DeHouwer@UGent.be

phone: 003292646445

fax: 003292646489 


\begin{abstract}
Instructions are known to have a profound impact on human behavior. Nevertheless, research on the effects of instructions is relatively scarce and scattered across different areas of research in psychology and neuroscience. The current issue of this journal contains six papers that review research on instructions in different research areas. In this introduction to the special section, we provide the outline of a framework that focuses on five components that can be varied in research on this topic (sender, message, receiver, context, and outcome). The framework brings order to the boundless potential variability in research on the effects of messages (i.e., it has heuristic value) and highlights that past research explored only a tiny fraction of what is possible (i.e., it has predictive value). Moreover, it reveals that research in different areas tends to examine different instantiations of the five components. The latter observation implies that much can be gained from closer interactions between researchers from different areas.
\end{abstract}

Keywords: Instructions; symbolic messages; framework 


\section{Toward a Unified Framework for Research on Instructions and other Messages: An Introduction to the Special Section on the Power of Instructions}

Instructions are symbolic messages that describe what one should do in a given situation. They typically consist of words but also pictures or even gestures can function as instructions. Hence, instructions from a subclass of symbolic messages that is delineated not by topographical characteristics but by content. In this paper, we do not discuss instruction in the educational sense (i.e., structured ways in which teachers educate pupils) but focus on the effects of instructions and other symbolic messages. It is difficult to overestimate the power of instructions. Just try to imagine a world in which humans are not able to generate or follow instructions. Losing the ability to act in line with instructions would greatly reduce the efficiency and efficacy of human action, eliminate a vital route for transmitting information within and between generations, and take away much of the competitive edge that humans have over other animals.

In light of the importance of instructions for human behavior, it is striking to see that both within neuroscience and psychology, there is relatively little research on how instructions and other symbolic messages guide behavior. Moreover, the studies that have been conducted are spread across various areas of research. For instance, cognitive psychologists have recently started to explore the (automatic) behavioral effects of instructed but never practiced stimulus-response mappings (e.g., "if you see the letter L, press a left key"; e.g., Liefooghe, Wenke, \& De Houwer, 2012; Meiran, Cole, \& Braver, 2012) whereas cognitive neuroscientists examined the brain activity that is associated with those effects (e.g., Cole, Laurent, \& Stocco, 2013). Independently, social and motivation psychologists studied the effects of so-called implementation intentions, which are in essence instructions on how to react in future situations (e.g., "the next time that I crave cigarettes, I will take a 
chewing gum instead"; Gollwitzer, 1999). In an entirely different domain of psychology, persuasion researchers examined the effects of instructions and other messages on attitudes (e.g., "This new soft-drink will help you feel rejuvenated"). Placebo researchers, on the other hand, looked at the health implications of messages about the expected outcomes of interventions (e.g., "Using this cream will reduce your pain"; Shapiro \& Shapiro, 1997). Finally, in relative isolation from all these areas of research, hypnosis researchers have examined specific ways of delivering instructions to specific subsets of people (i.e., people who are highly suggestible; Oakley \& Halligan, 2013). 1

Based on the arguments that we present later on in this paper, we believe that there is much to be gained from a closer interaction between researchers from different research areas who study different aspects of the effects of instructions and other messages. This realization encouraged Marcel Brass, Jan De Houwer, and Christian Büchel to organize the 27th Attention \& Performance meeting on the topic of the power of instructions. The meeting for the first time brought together researchers from different areas in psychology who study the effects of instructions and other messages. As a result of this meeting, Marcel Brass and Jan De Houwer were given the opportunity to edit the special section to which this paper provides an introduction. The other papers in this special section provide state-of-the-art reviews of research on the effects of instructions and other messages as they have been studied in a particular area of research. As such, this special section provides the impetus for closer interactions between researchers from different backgrounds who share their fascination for the power of instructions.

\footnotetext{
1 Unbeknownst to many cognitively inspired researchers, there is also extensive research on the effects of instructions (i.e., rule-governed behavior) within functional psychology, that is, the branch of psychology that focuses on the relations between environment and behavior (e.g., radical behavioris m as introduced by Skinner, 1938; and Contextual Behavioral Science; see Hayes, Barnes-Holmes, \& Wilson, 2012). For an overview of this research, please consult Hughes and Barnes-Holmes (2016) or O’Hora, Barnes-Holmes, \& Stewart (2014).
} 
Before we provide a summary of the various contributions to the special section, we sketch the outlines of a framework that highlights different ways in which the effects of instructions and other symbolic messages can be examined. As such, it not only provides a possible way to relate existing research in various research areas (i.e., its heuristic function) but also highlights new, previously unchartered ways of studying instructions (i.e., its predictive function). The framework (see Table 1) focuses on five components that can be varied when studying the effects of instructions and other messages: The nature of (1) the sender, (2) the message, (3) the receiver, (4) the context, and (5) the outcome that is examined (i.e., the dependent measure). Different studies, and more generally, different strands of research on the effects of messages, can be classified on the basis of the way in which the five components are instantiated. As a first sketch of the framework, in the next paragraph, we briefly describe a small subset of component values, that is, ways in which the five components of the framework have been instantiated in the past (also see Table 1).

First, various properties of the sender can and have been manipulated. For instance, persuasive messages tend to exert a stronger influence if the sender is perceived as highly credible (e.g., Vogel \& Wänke, 2016). Note that in some studies, the sender and the receiver are one and the same person, as is the case in studies on implementation intentions in which people instruct themselves (Gollwitzer, 1999). Second, one can vary numerous message properties. For instance, whereas instructions in a strict sense specify which actions should be undertaken to achieve a goal (e.g., "“if you see the letter L, press a left key”; e.g., Liefooghe et al., 2012; Meiran et al., 2012), other messages have less prescriptive connotations, such as messages about stimulus properties (e.g., "This soft-drink tastes like cherry"; Vogel \& Wänke, 2016). Because instructions and other messages are not necessarily verbal (i.e., do not necessarily involve words), the mode of the message (verbal or nonverbal) can also be 
varied. Third, the effects of messages can depend on multiple properties of the receiver. For instance, one crucial property is the language ability and learning history of the receiver. Another important property that has been studied extensively in research on hypnosis is the suggestibility of the receiver (e.g., Oakley \& Halligan, 2013). Fourth, instructions and other messages are always presented in a certain context. Hence, the properties of the context also have the potential to moderate the impact of those messages. For instance, social psychological research on obedience showed already many years ago that ethically questionable instructions (e.g., to administer an electric shock to another person) are more likely to be followed in the context of a lab setting at a university than in the context of a shabby office building (Milgram, 1974). Likewise, placebo effects vary dramatically as a function of the treatment context (e.g., the presence of diagnostic instruments or the appearance of the hospital room; Colloca \& Miller, 2011). Fifth, instructions and other messages can have many different outcomes. They can influence the speed and accuracy of motor behavior (e.g., Liefooghe et al., 2012; Meiran et al., 2012), brain activity (Cole et al., 2013) and even neural activity in the spinal cord (Eippert, Finsterbusch, Bingel, \& Büchel, 2009), physiological responses such as skin conductance (e.g., Raes, De Houwer, De Schryver, Brass, \& Kalisch, 2014) or bodily responses involved in diseases (Shapiro \& Shapiro, 1997), and emotional responses such as feelings towards brands or social groups (e.g., Vogel \& Wänke, 2016). Finally, the effect of each component value can interact with the value of one or more other components. For instance, persuasive messages have a much stronger effect when the sender and receiver share properties (e.g., Vogel \& Wänke, 2016).

When taking into account the fact that all five components in the framework can be varied independently or in various combinations, it becomes clear that the potential diversity of research on the effects of instructions and other messages is boundless. Past research 
explored only a tiny fraction of this potential diversity. The framework offers researchers a way to identify the regions they occupy within this space of possibilities and thus to relate themselves to and communicate more effectively with other researchers, including those who operate in different areas of psychology. As can be seen in Table 1, most strands of research focused on the effects of verbal messages that highly credible senders present in lab settings to receivers with good language abilities. However, the different strands of research also differ in important ways, most clearly with regard to the outcome that was examined and the content of the message. Because the framework shows that different strands occupy different regions within the realm of research on the effects of messages, it highlights the fact that researchers from one strand can look at research in other strands to gain knowledge and inspiration about potential moderators of the effects that they study. For instance, it might well be that known moderators of persuasion effects (e.g., credibility of the sender, similarity of sender and receiver) also moderate placebo effects or the effects of instructed S-R mappings on motor behavior. Likewise, the degree to which a receiver is susceptible to hypnotic suggestions might also moderate the efficacy of implementation intentions. Or the brain areas involved in the effects of instructed stimulus-response mappings might also be involved in implementation intentions. In sum, there is much to be gained from a closer interaction between researchers from different areas in psychology who study different aspects of the effects of messages.

In the final part of this paper, we provide a brief summary of the other contributions to the special section. Cole, Braver, and Meiran (this issue) not only provide an overview of research on the ability to follow new instructions immediately but also sketch a model of how such an implementation can be achieved at the neural level. Two aspects of rapid instructed task learning (RITL) are discussed: First, the paradox that RITL, which can be understood as 
a highly flexible process, at the same time creates inflexibility. Second, how RITL is achieved at the neurocognitive level, namely by prefrontal rerouting of perceptual-motor connections. This rerouting process is embedded in the flexible hub framework which assumes that there is global connectivity within fronto-parietal cognitive control networks and that connectivity patterns within $\mathrm{CCN}$ regions can flexibly change.

Brass, Liefooghe, Braem, and De Houwer (this issue) provide an overview of recent neurocognitive findings on instruction following. The main thesis of the paper states that there is a dissociation between 'knowing and doing' in instruction following. This dissociation refers to the observation that people are sometimes not able to follow verbal instructions even though they perfectly understand and remember these instructions. The paper summarizes evidence from different research domains that a declarative representation of the instruction needs to be transformed into a procedural representation to allow for its implementation. This transformation follows three different phases. In the instruction phase, the linguistic information is transformed into a hierarchical task model that consist of condition-action rules. In the implementation phase, the most relevant condition-action rules are put in a highly active state. Finally, in the application stage, the now relevant conditionaction rule is applied. It is argued that all three stages of instruction following rely to a varying degree on fronto-parietal network.

Koban, Jepma, Geuter, and Wager (this issue) provide an integrative perspective on different forms of socially-mediated information about pain and emotion. First, they review research on the impact of social observation on fear learning, pain processing, and reward learning. Second, they address the role of social influence and conformity. Third, they focus on social instructions such as placebo instructions and hypnosis within the context of research on fear and pain. Finally, they review the literature on the interaction between social 
information and experience within the context of appetitive and aversive learning. The authors also develop a model of how social information can influence processing pain and affective states. They assume that an instructed state representation is created in the prefrontal cortex and that this representation creates predictions about one's experiences. This top-down information will then alter stimulus processing but also appraisal processes in different parts of the brain.

Hommel and Colzato (this issue) focus on the topic of metacontrol, that is, the control of cognitive control. More specifically, they review the literature on how metacontrol can be transmitted at the social level. They discuss interindividual variability in metacontrol, as well as the genetic, cultural, and ontogenetic determinants of biases in metacontrol. Moreover, they provide a theoretical account of the impact of those determinants on metacontrol. Their paper thus sheds light on how more indirect ways of instructing (via culture, peers, and situational cues) can have a major impact on cognition and behavior.

Terhune, Cleeremans, Raz, and Lynn (this issue) provide an in depth scientific treatment of the topic of hypnosis. They not only clarify the nature of hypnosis but also discuss its clinical potential, the underlying neurocognitive mechanisms, and the relation with other phenomena such as agency, metacognition, cognitive control, and social cognition. As such, their paper provides an ideal introduction to the literature on hypnosis. Moreover, it sets the agenda for future research on this unique way of providing instructions.

Finally, Landry, Lifshitz, and Raz (this issue) provide an overview of the brain imaging literature on hypnosis. They contrast two views on the neural basis of hypnosis. One view assumes that hypnosis operates via top-down control involving cognitive control related brain regions. The other view assumes that hypnosis alters processing in brain regions involved in self-related processing. Furthermore, the authors discuss the role of inter-individual differences 
in the susceptibility to hypnosis and the role of the type of hypnotic induction. The metaanalysis that they report did not provide strong evidence for either of the two theories but did reveal reliable activation in a perception-related brain region.

In sum, we hope that the special section not only offers a useful summary of the present state of the relevant literatures but also provide an impetus for a more concerted effort to understand the power of instructions. 


\section{References}

Brass, M., Liefooghe, B., Braem, S., \& De Houwer, J. (this issue). Following new task instructions: Evidence for a dissociation between knowing and doing. Neuroscience and Biobehavioral Reviews.

Cole, M. W., Laurent, P., \& Stocco A. (2013). Rapid instructed task learning: A new window into the human brain's unique capacity for flexible cognitive control. Cognitive, Affective, \& Behavioral Neuroscience. 13, 1-22.

Cole, M. W., Braver, T. S., \& Merian, N (this issue). The task novelty paradox: Flexible control of inflexible neural pathways during rapid instructed task learning. Neuroscience and Biobehavioral Reviews.

Eippert, F., Finsterbusch, J., Bingel, U., \& Büchel, C. (2009). Direct evidence for spinal cord involvement in placebo analgesia. Science, 326, 404.

Gollwitzer, P. M. (1999). Implementation intentions: Strong effects of simple plans. American Psychologist, 54, 493-503.

Hayes, S.C., Barnes-Holmes, D., \& Wilson, K.G. (2012). Contextual behavioral science: Creating a science more adequate to the challenge of the human condition. Journal of Contextual Behavioral Science, 1, 1-16.

Hommel, B., \& Colzato, L. (this issue). Sharing metacontrol: Mechanisms underlying the interpersonal transfer of persistence and flexibility. Neuroscience and Biobehavioral Reviews.

Hughes, S., \& Barnes-Holmes, D. (2016). Relational Frame Theory: Implications for the Study of Human Language and Cognition. In S. Hayes, D. Barnes-Holmes, R. Zettle, and T. Biglan (Eds.), Handbook of Contextual Behavioral Science. New York: Wiley.

Koban, L., Jepma, M., Geuter, S. \& Wager, T. D. (this issue). What's in a word? How 
instructions, suggestions, and social information change pain and emotion. Neuroscience and Biobehavioral Reviews.

Landry, M., Lifshitz, M., \& Raz, A. (this issue). Brain correlates of hypnosis: A systematic review and meta-analytic exploration. Neuroscience and Biobehavioral Reviews.

Liefooghe, B., Wenke, D., \& De Houwer, J. (2012). Instruction-based task-rule congruency effects. Journal of Experimental Psychology: Learning, Memory, \& Cognition, 38, 1325-1335.

Meiran, N., Cole, M. W., \& Braver, T. S. (2012). When planning results in loss of control: intention-based reflexivity and working-memory. Frontiers in Human Neuroscience, $6,104$.

Milgram, S. (1974). Obedience to authority: An experimental view. New York: Harper \& Row.

Oakley, D. A., \& Halligan, P. W. (2013). Hypnotic suggestion: Opportunities for cognitive neuroscience. Nature Reviews Neuroscience, 14, 565-576.

O’Hora, D., Barnes-Holmes, D., \& Stewart, I. (2014). Antecedent and consequential control of derived instruction-following. Journal of the Experimental Analysis of Behavior, 102(1), 66-85.

Price, D. D., Finniss, D. B., \& Benedetti, F. (2008). A comprehensive review of the placebo effect: recent advances and current thought. Annual Review of Psychology, 59, 56590.

Raes, A. K., De Houwer, J., De Schryver, M., Brass, M., \& Kalisch, R. (2014). Do CS-US pairings actually matter? A within-subject comparison of instructed fear conditioning with and without actual CS-US pairings. PLOS ONE 9(1): e84888.

Shapiro, A.K., \& Shapiro, E. (1997). The Powerful Placebo. From Ancient Priest to Modern 
Physician. Baltimore: Johns Hopkins University Press.

Skinner, B.F. (1938). The Behavior of Organisms: An Experimental Analysis. New York: Appleton-Century.

Terhune, D., Cleeremans, A., Raz, A., \& Lynn, S. J. (this issue). Hypnosis and top-down regulation of consciousness. Neuroscience and Biobehavioral Reviews.

Vogel, T., \& Wänke, M. (2016). Attitudes and attitude change (2nd ed.). NY:

Routledge/Taylor \& Francis Group. 


\section{Author Note}

Jan De Houwer, Sean Hughes, and Marcel Brass, Ghent University, Ghent, Belgium. The preparation of this paper was made possible by Methusalem Grant BOF16/MET_V/002 of Ghent University and the Interuniversity Attraction Poles Program initiated by the Belgian Science Policy Office (IUAPVII/33). Correspondence should be addressed to Jan De Houwer, Ghent University, Henri Dunantlaan 2, B-9000 Ghent, Belgium. Electronic mail can be sent to Jan.DeHouwer@UGent.be . 
Research Area

$\mathrm{CP} \quad \mathrm{CN} \quad \mathrm{II} \quad \mathrm{PE} \quad \mathrm{PL} \quad \mathrm{H}$

Component

Sender

$\begin{array}{llllllll}\text { High credibility } & X & X & X & X & X & X\end{array}$

Low credibility $\quad \mathrm{X}$

$\begin{array}{lllllll}\text { Other than receiver } & X & X & X & X & \text { X }\end{array}$

Same as receiver $\quad \mathrm{X} \quad \mathrm{X}$

Message

$\begin{array}{lllllll}\text { Content: Instruction } & \text { X } & \text { X } & \text { X } & \text { X } & \text { X } & \text { X }\end{array}$

$\begin{array}{lllll}\text { Content: Stimulus Properties } & \text { X } & \text { X } & \text { X }\end{array}$

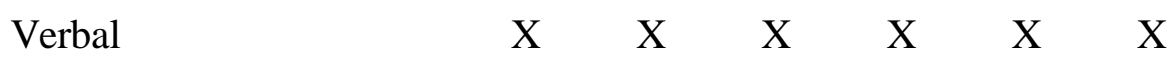

Nonverbal $\quad$ X

Receiver

$\begin{array}{llllllll}\text { Good language ability } & X & X & X & X & X & \text { X }\end{array}$

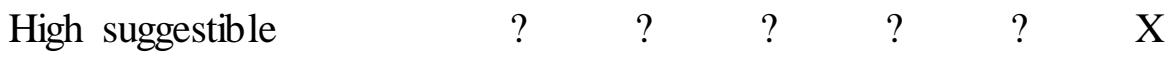

Low suggestible ? ? ? ? ?

Context

$\begin{array}{llllllll}\text { Lab setting } & X & X & X & X & X & \text { X }\end{array}$

$\begin{array}{lllll}\text { Real life context } & X & X & X & \text { X }\end{array}$

Outcome

Motor behavior

X $\quad X$

X

Brain activity

X

X

Physiology

X

Emotion

X $\quad$ X $\quad$ X 
Note. $C P=$ Cognitive Psychology; $C N=$ Cognitive Neuroscience $; I I=$ Implementation

Intentions; $P E=$ Persuasion; $P L=$ Placebo $; H=$ Hypnosis; $X=$ component value often instantiated in studies within that research area; ? = Unknown whether this component value is instantiated in studies within that research area. Note that the table presents only a tiny fraction of the possible values of the five components and that interactions between one or more components can in principle also be examined. 